\title{
AN ANALYSIS OF HDG METHODS FOR THE VORTICITY-VELOCITY-PRESSURE FORMULATION OF THE STOKES PROBLEM IN THREE DIMENSIONS
}

\author{
BERNARDO COCKBURN AND JINTAO CUI
}

\begin{abstract}
We provide the first a priori error analysis of a hybridizable discontinuous Galerkin (HDG) method for solving the vorticity-velocity-pressure formulation of the three-dimensional Stokes equations of incompressible fluid flow. By using a projection-based approach, we prove that, when all the unknowns use polynomials of degree $k \geq 0$, the $L^{2}$-norm of the errors in the approximate vorticity and pressure converge to zero with order $k+1 / 2$, whereas the error in the approximate velocity converges with order $k+1$.
\end{abstract}

\section{INTRODUCTION}

In this paper we provide the first a priori error analysis of the hybridizable discontinuous Galerkin (HDG) method proposed in 7 for solving the vorticityvelocity-pressure formulation of the Stokes equations of incompressible fluid flow, namely,

$$
\begin{array}{rlrl}
\boldsymbol{w}-\nabla \times \boldsymbol{u} & =0 & & \text { in } \Omega, \\
\nabla \times \boldsymbol{w}+\nabla p & =\boldsymbol{f} & & \text { in } \Omega, \\
\nabla \cdot \boldsymbol{u} & =0 & & \text { in } \Omega, \\
\boldsymbol{u} & =\boldsymbol{g} & & \text { on } \partial \Omega, \\
\int_{\Omega} p & =0 . &
\end{array}
$$

Here $\int_{\partial \Omega} \boldsymbol{g} \cdot \boldsymbol{n}=0$, and $\Omega \subset \mathbb{R}^{3}$ is a Lipschitz polyhedron.

To describe our results, let us briefly describe the evolution of HDG methods for incompressible fluid flow. Originally, the motivation for using hybridization techniques for finite elements for incompressible flow stemmed from the fact that the construction of finite dimensional spaces of divergence-free approximate velocities is extremely difficult. The construction of such spaces was undertaken back in 1972 [15], in 1979 [17, and in 1981 [25, 19]. However, the extension of these constructions to spaces of piecewise polynomials of arbitrary degree has been a long standing question. Later, in 24] it was shown that, in the two-dimensional case, spaces of divergence-free velocities can be systematically constructed provided polynomials of degree bigger than four are used. Lower order polynomial spaces with this

Received by the editor March 8, 2011 and, in revised form, May 25, 2011.

2010 Mathematics Subject Classification. Primary 65M60, 65N30, 35L65.

Key words and phrases. Discontinuous Galerkin methods, hybridization, incompressible fluid flow.

The first author was partially supported by the National Science Foundation (Grant DMS0712955) and by the Minnesota Supercomputing Institute.

(C)2011 American Mathematical Society Reverts to public domain 28 years from publication 
property do not exist, as was proven in 1975 in [16]. Extensions of these results to the three-dimensional case still remain an open problem. See the discussions in [3, 11, 13.

Only recently, two new approaches for generating divergence-free velocity approximations have emerged, namely, by postprocessing and by hybridization; see the discussion in [4. In the first approach, the approximate solution provided by a DG method is postprocessed in an element-by-element fashion to give rise to a new divergence-free approximate velocity. The first postprocessing technique of this type was introduced in the framework of DG methods for diffusion problems in 2]. In the framework of incompressible fluid flow, this was done for the first time in 2005 in [10; see also [12. Therein it was shown that, when the postprocessing becomes the identity, the DG method automatically provides a solenoidal velocity; this idea was developed in 2007 in [11] and [26]. This postprocessing can be easily applied to the previously introduced DG methods using elementwise divergence-free velocities proposed in 1990 in [1] and then in 1998 in [18].

In the second approach, the method is rewritten in an equivalent form in two steps. In the first, the continuity constraint on the normal components of the approximate velocities on the interelement boundaries is relaxed so that we now work with spaces of completely discontinuous velocities. In the second, the continuity of the normal component of the approximate velocity is imposed by a suitable set of equations. In this manner, the construction of divergence-free velocity spaces is completely avoided. This was done for the first time in 2006 in 3] for a DG method and then in 2005 in [5, 6] for a mixed method. Both of these methods were based on vorticity-velocity-pressure formulations of the Stokes equations.

Recently, a new HDG method, based on a velocity gradient-velocity-pressure formulation and using spaces of exactly divergence-free approximate velocities was introduced and analyzed in [13]. All the components of the approximate solution, which use polynomial spaces of degree $k$, were proven to converge with the optimal order of $k+1$ in $L^{2}$-norm for any $k \geq 0$. Moreover, an element-by-element postprocessed velocity approximation, which is divergence-conforming and exactly divergence-free, was shown to converge with order $k+2$ for $k \geq 1$ and with order 1 for $k=0$. These HDG method can be obtained as limits of the HDG methods introduced in 2010 in [23] and share with them all the above-mentioned convergence properties; see 8 .

Similar HDG methods based on a vorticity-velocity-pressure formulation had been previously introduced in 2009 in [7, but have not been theoretically analyzed. They had only been experimentally compared with those based on a velocity gradient-velocity-pressure formulation in 22] for the two-dimensional case. Here, we provide the first a priori error analysis of the HDG methods proposed in [7] for the three-dimensional case.

The idea of the analysis in this paper is similar to that of the HDG methods based on the velocity gradient-velocity-pressure formulation [8]. Indeed, it consists of estimating a projection of the errors that is tailored to the very structure of the numerical traces of the method. However, unlike the projection used in [8, the velocity and pressure components of the projection are decoupled from the vorticity component. In fact, the projection for the velocity and pressure is the one used in the analysis of HDG methods for diffusion problems in [9], whereas the projection for the vorticity is nothing but the standard $L^{2}$-projection. We show that the 
approximated vorticity and pressure, which are polynomials of degree $k$, converge with order $k+1 / 2$ in $L^{2}$-norm for any $k \geq 0$. Moreover, the approximated velocity converges with order $k+1$.

Finally, let us briefly compare these results with those associated with edge elements. In an early paper [20, the first-type Nédélec edge elements and continuous piecewise polynomial of order $k$ were used for the approximate velocity and pressure, respectively. Both components were proven to converge with a suboptimal order of $k$ in the energy norm for any $k \geq 0$. Later, in 21, the $H$ (curl)-conforming (second-type) edge elements of order $k$ were used to approximate the vorticity, and the $H$ (div)-conforming edge elements of order $k-1$ is applied to approximate the velocity. It was shown that both vorticity and velocity converge with order $k-1$ in $L^{2}$-norm for $k \geq 1$.

The paper is organized as follows. In Section 2, we present the method and state and discuss our main results. In Section 3, we provide a detailed proof of our error estimates. We end in Section 4 with some concluding remarks.

\section{MAIN RESULTS}

2.1. The HDG method. Let $\mathcal{T}_{h}$ be a shape-regular triangulation of $\Omega$ which consists of tetrahedra $T$. We denote by $\varepsilon_{h}$ the set of all faces $F$ of all tetrahedra $T$ of the triangulation $\mathcal{T}_{h}$ and by $\partial \Omega_{h}$ the set of boundaries $\partial T$ of the elements $T$ of $\mathcal{T}_{h}$.

The HDG method seeks an approximation of the solution $\left(\left.\boldsymbol{w}\right|_{\Omega},\left.\boldsymbol{u}\right|_{\Omega},\left.p\right|_{\Omega},\left.\boldsymbol{u}\right|_{\mathcal{E}_{h}}\right)$ of the problem (1.1),$\left(\boldsymbol{w}_{h}, \boldsymbol{u}_{h}, p_{h}, \widehat{\boldsymbol{u}}_{h}\right)$, in the space $\boldsymbol{W}_{h} \times \boldsymbol{V}_{h} \times P_{h} \times \boldsymbol{M}_{h}$, where

$$
\begin{aligned}
& \boldsymbol{W}_{h}:=\left\{\boldsymbol{w} \in \boldsymbol{L}_{2}\left(\mathcal{T}_{h}\right):\left.\boldsymbol{w}\right|_{T} \in \boldsymbol{P}_{k}(T) \quad \forall T \in \mathcal{T}_{h}\right\}, \\
& \boldsymbol{V}_{h}:=\left\{\boldsymbol{v} \in \boldsymbol{L}_{2}\left(\mathcal{T}_{h}\right):\left.\boldsymbol{v}\right|_{T} \in \boldsymbol{P}_{k}(T) \quad \forall T \in \mathcal{T}_{h}\right\}, \\
& P_{h}:=\left\{q \in L_{2}\left(\mathcal{T}_{h}\right):\left.q\right|_{T} \in P_{k}(T) \quad \forall T \in \mathcal{T}_{h}\right\}, \\
& \boldsymbol{M}_{h}:=\left\{\boldsymbol{\mu} \in \boldsymbol{L}_{2}\left(\mathcal{E}_{h}\right):\left.\boldsymbol{\mu}\right|_{T} \in \boldsymbol{P}_{k}(F) \quad \forall F \in \mathcal{E}_{h}\right\} \text {, }
\end{aligned}
$$

$P_{k}(T)$ is the space of polynomials of total degree at most $k$ defined on $T$, and $\boldsymbol{P}_{k}(T)=\left[P_{k}(T)\right]^{n}$. The approximation is defined by requiring that

$$
\begin{aligned}
\left(\boldsymbol{w}_{h}, \boldsymbol{\tau}\right)_{\mathcal{T}_{h}}-\left(\boldsymbol{u}_{h}, \nabla \times \boldsymbol{\tau}\right)_{\mathcal{T}_{h}}-\left\langle\widehat{\boldsymbol{u}}_{h}, \boldsymbol{\tau} \times \boldsymbol{n}\right\rangle_{\partial \mathcal{T}_{h}} & =0, \\
\left(\boldsymbol{w}_{h}, \nabla \times \boldsymbol{v}\right)_{\mathcal{T}_{h}}-\left(p_{h}, \nabla \cdot \boldsymbol{v}\right)_{\mathcal{T}_{h}}+\left\langle\boldsymbol{n} \times \widehat{\boldsymbol{w}}_{h}+\widehat{p}_{h} \boldsymbol{n}, \boldsymbol{v}\right\rangle_{\partial \mathcal{T}_{h}} & =(\boldsymbol{f}, \boldsymbol{v})_{\mathcal{T}_{h}}, \\
-\left(\boldsymbol{u}_{h}, \nabla q\right)_{\mathcal{T}_{h}}+\left\langle\widehat{\boldsymbol{u}}_{h}, q \boldsymbol{n}\right\rangle_{\partial \mathcal{T}_{h}} & =0, \\
\left\langle\boldsymbol{n} \times \widehat{\boldsymbol{w}}_{h}+\widehat{p}_{h} \boldsymbol{n}, \boldsymbol{\mu}\right\rangle_{\partial \mathcal{T}_{h} \backslash \partial \Omega} & =0, \\
\left\langle\widehat{\boldsymbol{u}}_{h}, \boldsymbol{\mu}\right\rangle_{\partial \Omega} & =\langle\boldsymbol{g}, \boldsymbol{\mu}\rangle_{\partial \Omega}, \\
\left(p_{h}, 1\right)_{\mathcal{T}_{h}} & =0,
\end{aligned}
$$

for all $(\boldsymbol{\tau}, \boldsymbol{v}, q, \boldsymbol{\mu}) \in \boldsymbol{W}_{h} \times \boldsymbol{V}_{h} \times P_{h} \times \boldsymbol{M}_{h}$, where

$$
\begin{aligned}
\widehat{\boldsymbol{w}}_{h} & =\boldsymbol{w}_{h}+\tau_{t}\left(\boldsymbol{u}_{h}-\widehat{\boldsymbol{u}}_{h}\right) \times \boldsymbol{n} & & \text { on } \partial \mathcal{T}_{h}, \\
\widehat{p}_{h} & =p_{h}+\tau_{h}\left(\boldsymbol{u}_{h}-\widehat{\boldsymbol{u}}_{h}\right) \cdot \boldsymbol{n} & & \text { on } \partial \mathcal{T}_{h} .
\end{aligned}
$$

Here the stabilization functions $\tau_{t}$ and $\tau_{n}$ are taken to be constants on each face on $\partial \mathcal{T}_{h}$. 
Note that we have used the following notation. We write

$$
(v, w)_{\mathcal{T}_{h}}=\sum_{T \in \mathcal{T}_{h}}(v, w)_{T} \quad \text { and } \quad(\boldsymbol{v}, \boldsymbol{w})_{\mathcal{T}_{h}}=\sum_{i=1}^{3}\left(v_{i}, w_{i}\right)_{\mathcal{T}_{h}},
$$

and

$$
\langle v, w\rangle_{\partial \mathcal{T}_{h}}=\sum_{T \in \mathcal{T}_{h}}\langle v, w\rangle_{\partial T} \quad \text { and } \quad\langle\boldsymbol{v}, \boldsymbol{w}\rangle_{\partial \mathcal{T}_{h}}=\sum_{i=1}^{3}\left\langle v_{i}, w_{i}\right\rangle_{\partial \mathcal{T}_{h}} .
$$

Here, $(\cdot, \cdot)_{T}$ and $\langle\cdot, \cdot\rangle_{\partial T}$ are the inner products of $L^{2}(T)$ and $L^{2}(\partial T)$, respectively.

2.2. The projection. From here on we denote by $\|v\|_{H^{\ell}(T)}$ the usual $H^{\ell}$-norm of $v$ on the domain $T$. We set $\boldsymbol{H}^{\ell}:=\left[H^{\ell}(T)\right]^{n}$ and $\|\boldsymbol{v}\|_{\boldsymbol{H}^{\ell}(T)}:=\sum_{i=1}^{n}\left\|v_{i}\right\|_{H^{\ell}(T)}$. When $\ell=0$, we simply write $\|\boldsymbol{v}\|_{D}$ instead of $\|\boldsymbol{v}\|_{\boldsymbol{H}^{0}(D)} \equiv\|\boldsymbol{v}\|_{L^{2}(D)}$.

Next, we introduce the projection we are going to use to carry out our analysis. Given a function $(\boldsymbol{w}, \boldsymbol{u}, p)$ in $\boldsymbol{H}^{1}\left(\mathcal{T}_{h}\right) \times \boldsymbol{H}^{1}\left(\mathcal{T}_{h}\right) \times H^{1}\left(\mathcal{T}_{h}\right)$, we define its projection $(\boldsymbol{\Pi} \boldsymbol{w}, \boldsymbol{\Pi} \boldsymbol{u}, \Pi p)$ as follows. On an arbitrary element $T$ of the triangulation $\mathcal{T}_{h}$, we require that

$$
\begin{aligned}
(\boldsymbol{\Pi} \boldsymbol{w}-\boldsymbol{w}, \boldsymbol{\tau})_{T} & =0 & & \forall \boldsymbol{\tau} \in \boldsymbol{P}_{k}(T), \\
(\boldsymbol{\Pi} \boldsymbol{u}-\boldsymbol{u}, \boldsymbol{v})_{T} & =0 & & \forall \boldsymbol{v} \in \boldsymbol{P}_{k-1}(T), \\
(\Pi p-p, q)_{T} & =0 & & \forall q \in P_{k-1}(T), \\
\left\langle\Pi p-p+\tau_{n}(\boldsymbol{\Pi} \boldsymbol{u}-\boldsymbol{u}) \cdot \boldsymbol{n}, \mu\right\rangle_{F} & =0 & & \forall \mu \in P_{k}(F),
\end{aligned}
$$

for all faces $F$ of the tetrahedron $T$.

We see that the $\boldsymbol{\Pi} \boldsymbol{w}$ is the simple $L^{2}$-projection of $\boldsymbol{w}$ into $\boldsymbol{P}_{k}(T)$. The component $(\boldsymbol{\Pi} \boldsymbol{u}, \Pi p)$ is nothing but the projection used in the analysis of HDG methods for diffusion problems in [9] with the stabilization parameter $\tau$ used therein replaced by $1 / \tau_{n}$. As a consequence, we have the following result.

Theorem 2.1 (9]). Suppose that $k \geq 0$ and that $\left.\tau_{n}\right|_{\partial T}>0$. Then the system (2.4b), (2.4c) and (2.4d) is uniquely solvable for $\Pi \boldsymbol{u}$ and $\Pi p$. Furthermore, when $\nabla \cdot \boldsymbol{u}=0$, there is a constant $C$ independent of $T$ and $\tau_{n}$ such that

$$
\begin{aligned}
\|\boldsymbol{\Pi} \boldsymbol{u}-\boldsymbol{u}\|_{T} & \leq C h_{T}^{\ell_{u}+1}|\boldsymbol{u}|_{\boldsymbol{H}^{\ell_{u}+1}(T)}+C \frac{h_{T}^{\ell_{p}+1}}{\left(\tau_{n}\right)_{T}^{*}}|p|_{H^{\ell_{p}+1}(T)}, \\
\|\Pi p-p\|_{T} & \leq C h_{T}^{\ell_{p}+1}|p|_{H^{\ell_{p}+1}(T)},
\end{aligned}
$$

for $\ell_{p}, \ell_{\boldsymbol{u}}$ in $[0, k]$. Here $\left(\tau_{n}\right)_{T}^{*}:=\left.\min \tau_{n}\right|_{\partial T \backslash F^{*}}$, where $F^{*}$ is a face of $T$ at which $\left.\tau_{n}\right|_{\partial T}$ is minimum.

2.3. The a priori error analysis. We first introduce the following dual problem. For any given $\boldsymbol{\theta} \in \boldsymbol{L}_{2}(\Omega)$, let $(\boldsymbol{\psi}, \boldsymbol{\phi}, \phi)$ be the solution of

$$
\begin{aligned}
& \psi+\nabla \times \phi=0 \quad \text { in } \Omega, \\
& -\nabla \times \boldsymbol{\psi}-\nabla \phi=\boldsymbol{\theta} \quad \text { in } \Omega \text {, } \\
& -\nabla \cdot \phi=0 \quad \text { in } \Omega \text {, } \\
& \phi=\mathbf{0} \quad \text { on } \partial \Omega, \\
& \int_{\Omega} \phi=0 \text {. }
\end{aligned}
$$


We assume that, for some real number $s$, we have

$$
\|\boldsymbol{\psi}\|_{H^{s+1}(\Omega)}+\|\boldsymbol{\phi}\|_{H^{s+2}(\Omega)}+\|\phi\|_{H^{s+1}(\Omega)} \leq C\|\boldsymbol{\theta}\|_{H^{s}(\Omega)} .
$$

In the three-dimensional case, we have that $s \leq 0$ if the domain is convex (cf. [14]).

We are now ready to state our main results, which give estimates of the projection of the approximation errors, namely, $\boldsymbol{\epsilon}^{\boldsymbol{w}}:=\boldsymbol{\Pi} \boldsymbol{w}-\boldsymbol{w}_{h}, \boldsymbol{\epsilon}^{\boldsymbol{u}}:=\boldsymbol{\Pi} \boldsymbol{u}-\boldsymbol{u}_{h}, \epsilon^{p}:=\Pi p-p_{h}$ and $\boldsymbol{\epsilon}^{\widehat{u}}:=\boldsymbol{P}_{\partial} \boldsymbol{u}-\widehat{\boldsymbol{u}}_{h}$. Here, $\boldsymbol{P}_{\partial}$ is the $L_{2}$-projection into $\boldsymbol{M}_{h}$. They are going to be stated in terms of the total average of the projection of the error in the pressure over $\Omega$ which we denote by

$$
\overline{\Pi p-p}=\frac{1}{|\Omega|} \int_{\Omega}(\Pi p-p) d x,
$$

and in terms of the quantity

$$
\|(\boldsymbol{\Pi} \boldsymbol{w}-\boldsymbol{w}, \boldsymbol{\Pi} \boldsymbol{u}-\boldsymbol{u})\|_{\tau_{t}, \partial \mathcal{T}_{h}}:=\left\|\tau_{t}^{-1 / 2} \boldsymbol{n} \times(\boldsymbol{\Pi} \boldsymbol{w}-\boldsymbol{w})+\tau_{t}^{1 / 2}(\boldsymbol{\Pi} \boldsymbol{u}-\boldsymbol{u})_{t}\right\|_{\partial \mathcal{T}_{h}},
$$

which can be easily estimated by means of the following simple result whose proof is presented in the Appendix.

Proposition 2.2. For all, $(\boldsymbol{\zeta}, \boldsymbol{\eta}) \in \boldsymbol{H}^{1}\left(\mathcal{T}_{h}\right) \times \boldsymbol{H}^{1}\left(\mathcal{T}_{h}\right)$, we have

$$
\begin{aligned}
\|(\boldsymbol{\Pi} \boldsymbol{\zeta}-\boldsymbol{\zeta}, \boldsymbol{\Pi} \boldsymbol{\eta}-\boldsymbol{\eta})\|_{\tau_{t}, \partial \mathcal{T}_{h}} \leq & C \max _{K \in \mathcal{T}_{h}} h_{T}^{1 / 2}\left\|\tau_{t}^{-1}\right\|_{L^{\infty}(\partial T)}^{1 / 2} E(\boldsymbol{\Pi} \boldsymbol{\zeta}, \boldsymbol{\zeta}) \\
& +C \max _{K \in \mathcal{T}_{h}} h_{T}^{1 / 2}\left\|\tau_{t}\right\|_{L^{\infty}(\partial T)}^{1 / 2} E(\boldsymbol{\Pi} \boldsymbol{\eta}, \boldsymbol{\eta}),
\end{aligned}
$$

where

$$
E^{2}(\boldsymbol{\Theta}, \boldsymbol{\theta}):=\inf _{\boldsymbol{S} \in \boldsymbol{W}_{h}} \sum_{K \in \mathcal{T}_{h}}\left(h_{T}^{-2}\left(\|\boldsymbol{\Theta}-\boldsymbol{\theta}\|_{T}^{2}+\|\boldsymbol{S}-\boldsymbol{\theta}\|_{T}^{2}\right)+\|\nabla(\boldsymbol{S}-\boldsymbol{\theta})\|_{T}^{2}\right),
$$

and $C$ is a constant depending on the shape-regularity constant of the elements and on the polynomial degree $k$.

Theorem 2.3. Suppose that $k \geq 0$ and that $\tau_{n}, \tau_{t}>0$ on $\partial \mathcal{T}_{h}$. Then we have

$$
\begin{aligned}
\left\|\boldsymbol{\epsilon}^{\boldsymbol{w}}\right\|_{\Omega} & \leq\left\|\left(\boldsymbol{\Pi} \boldsymbol{w}-\boldsymbol{w}, \boldsymbol{\Pi} \boldsymbol{u}-\boldsymbol{P}_{\partial} \boldsymbol{u}\right)\right\|_{\tau_{t}, \partial \mathcal{T}_{h}}, \\
\left\|\epsilon^{p}\right\|_{\Omega} & \leq \overline{(\Pi p-p)}|\Omega|^{1 / 2}+C_{\tau_{t}}\left\|\left(\boldsymbol{\Pi} \boldsymbol{w}-\boldsymbol{w}, \boldsymbol{\Pi} \boldsymbol{u}-\boldsymbol{P}_{\partial} \boldsymbol{u}\right)\right\|_{\tau_{t}, \partial \mathcal{T}_{h}},
\end{aligned}
$$

where

$$
C_{\tau_{t}}:=1+\left(\max _{T \in \mathcal{T}_{h}} h_{T}\left\|\tau_{t}\right\|_{L^{\infty}(\partial T}\right)^{1 / 2} .
$$

Moreover, if the elliptic regularity inequality (2.6) holds with $s=0$, we have

$$
\left\|\boldsymbol{\epsilon}^{\boldsymbol{u}}\right\|_{\Omega} \leq C H_{\tau}\left\|\left(\boldsymbol{\Pi} \boldsymbol{w}-\boldsymbol{w}, \boldsymbol{\Pi} \boldsymbol{u}-\boldsymbol{P}_{\partial} \boldsymbol{u}\right)\right\|_{\tau_{t}, \partial \mathcal{T}_{h}},
$$

where

$$
\begin{aligned}
H_{\tau}:= & \max _{T \in \mathcal{T}_{h}} h_{T}^{1 / 2}\left\|\tau_{t}^{-1}\right\|_{L^{\infty}(\partial T)}^{1 / 2} \\
& +\max _{T \in \mathcal{T}_{h}} h_{T}^{1 / 2}\left\|\tau_{t}\right\|_{L^{\infty}(\partial T)}^{1 / 2}\left(\max _{T \in \mathcal{T}_{h}} h_{T}^{\min \{1, k\}}+\max _{T \in \mathcal{T}_{h}}\left\|\left(\tau_{n}^{*}\right)^{-1}\right\|_{L^{\infty}(\partial T)}\right) .
\end{aligned}
$$

Combining the above result with Proposition 2.2, the approximation properties of the projection $(\boldsymbol{\Pi}, \Pi)$ (see Theorem 2.1) and those of the $L^{2}$-projection $\boldsymbol{\Pi}$, we 
immediately obtain the errors we sought. Thus, under the hypotheses of Theorem 2.3. and when the solution is very smooth, we have that

$$
\begin{aligned}
\left\|\boldsymbol{w}-\boldsymbol{w}_{h}\right\|_{\Omega} & \leq C h^{k+1 / 2}, \\
\left\|p-p_{h}\right\|_{\Omega} & \leq C h^{k+1 / 2}, \\
\left\|\boldsymbol{u}-\boldsymbol{u}_{h}\right\|_{\Omega} & \leq C h^{k+1},
\end{aligned}
$$

provided $\tau_{t}, 1 / \tau_{t}$ and $1 / \tau_{n}^{*}$ remain of order one on $\partial \mathcal{T}_{h}$. Thus, we see that the approximate vorticity and pressure converge with an order which is suboptimal by $1 / 2$, whereas the velocity converges with optimal order.

\section{PROOFS}

We denote $\boldsymbol{S}_{\tau} \boldsymbol{\mu}:=\tau_{t} \boldsymbol{n} \times \boldsymbol{\mu} \times \boldsymbol{n}+\tau_{n} \boldsymbol{\mu} \cdot \boldsymbol{n} \boldsymbol{n}$, and $\boldsymbol{v}_{t}:=\boldsymbol{v}-(\boldsymbol{v} \cdot \boldsymbol{n}) \boldsymbol{n}=\boldsymbol{n} \times \boldsymbol{v} \times \boldsymbol{n}$.

3.1. The equations satisfied by the projection of the errors. We begin by obtaining the equations satisfied by the projection of the errors.

Lemma 3.1. We have

$$
\begin{aligned}
\left(\boldsymbol{\epsilon}^{\boldsymbol{w}}, \boldsymbol{\tau}\right)_{\mathcal{T}_{h}}-\left(\boldsymbol{\epsilon}^{\boldsymbol{u}}, \nabla \times \boldsymbol{\tau}\right)_{\mathcal{T}_{h}}-\left\langle\boldsymbol{\epsilon}^{\widehat{u}}, \boldsymbol{\tau} \times \boldsymbol{n}\right\rangle_{\partial \mathcal{T}_{h}} & =0 \\
\left(\boldsymbol{\epsilon}^{\boldsymbol{w}}, \nabla \times \boldsymbol{v}\right)_{\mathcal{T}_{h}}-\left(\boldsymbol{\epsilon}^{p}, \nabla \cdot \boldsymbol{v}\right)_{\mathcal{T}_{h}} & \\
+\left\langle\boldsymbol{n} \times \boldsymbol{\epsilon}^{\boldsymbol{w}}+\boldsymbol{\epsilon}^{p} \boldsymbol{n}+\boldsymbol{S}_{\tau}\left(\boldsymbol{\epsilon}^{\boldsymbol{u}}-\boldsymbol{\epsilon}^{\widehat{\boldsymbol{u}}}\right), \boldsymbol{v}\right\rangle_{\partial \mathcal{T}_{h}} & = \\
\langle\boldsymbol{n} \times(\boldsymbol{\Pi} \boldsymbol{w}-\boldsymbol{w}) & \left.+\boldsymbol{S}_{\tau}\left(\boldsymbol{\Pi} \boldsymbol{u}-\boldsymbol{P}_{\partial} \boldsymbol{u}\right), \boldsymbol{v}_{t}\right\rangle_{\partial \mathcal{T}_{h}}, \\
-\left(\boldsymbol{\epsilon}^{\boldsymbol{u}}, \nabla q\right)_{\mathcal{T}_{h}}+\left\langle\boldsymbol{\epsilon}_{h}^{\widehat{u}}, q \boldsymbol{n}\right\rangle_{\partial \mathcal{T}_{h}} & =0 \\
\left\langle\boldsymbol{n} \times \boldsymbol{\epsilon}^{\boldsymbol{w}}+\boldsymbol{\epsilon}^{p} \boldsymbol{n}+\boldsymbol{S}_{\tau}\left(\boldsymbol{\epsilon}^{\boldsymbol{u}}-\boldsymbol{\epsilon}^{\widehat{\boldsymbol{u}}}\right), \boldsymbol{\mu}\right\rangle_{\partial \mathcal{T}_{h} \backslash \partial \Omega} & = \\
\langle\boldsymbol{n} \times(\boldsymbol{\Pi} \boldsymbol{w}-\boldsymbol{w}) & \left.+\boldsymbol{S}_{\tau}\left(\boldsymbol{\Pi} \boldsymbol{u}-\boldsymbol{P}_{\partial} \boldsymbol{u}\right), \boldsymbol{\mu}_{t}\right\rangle_{\partial \mathcal{T}_{h} \backslash \partial \Omega}, \\
\left\langle\boldsymbol{\epsilon}^{\widehat{u}}, \boldsymbol{\mu}\right\rangle_{\partial \Omega} & =0 \\
\left(\epsilon^{p}, 1\right)_{\mathcal{T}_{h}} & =(\Pi p-p, 1)_{\mathcal{T}_{h}},
\end{aligned}
$$

for all $(\boldsymbol{\tau}, \boldsymbol{v}, q, \boldsymbol{\mu}) \in \boldsymbol{W}_{h} \times \boldsymbol{V}_{h} \times P_{h} \times \boldsymbol{M}_{h}$.

Proof. Note that the exact solution satisfies the following equations:

$$
\begin{aligned}
(\boldsymbol{w}, \boldsymbol{\tau})_{\mathcal{T}_{h}}-(\boldsymbol{u}, \nabla \times \boldsymbol{\tau})_{\mathcal{T}_{h}}-\langle\boldsymbol{u}, \boldsymbol{\tau} \times \boldsymbol{n}\rangle_{\partial \mathcal{T}_{h}} & =0, \\
(\boldsymbol{w}, \nabla \times \boldsymbol{v})_{\mathcal{T}_{h}}-(p, \nabla \cdot \boldsymbol{v})_{\mathcal{T}_{h}}+\langle\boldsymbol{n} \times \boldsymbol{w}+p \boldsymbol{n}, \boldsymbol{v}\rangle_{\partial \mathcal{T}_{h}} & =(\boldsymbol{f}, \boldsymbol{v})_{\mathcal{T}_{h}}, \\
-(\boldsymbol{u}, \nabla q)_{\mathcal{T}_{h}}+\langle\boldsymbol{u}, q \boldsymbol{n}\rangle_{\partial \mathcal{T}_{h}} & =0, \\
\langle\boldsymbol{n} \times \boldsymbol{w}+p \boldsymbol{n}, \boldsymbol{\mu}\rangle_{\partial \mathcal{T}_{h} \backslash \partial \Omega} & =0, \\
\langle\boldsymbol{u}, \boldsymbol{\mu}\rangle_{\partial \Omega} & =\langle\boldsymbol{g}, \boldsymbol{\mu}\rangle_{\partial \Omega}, \\
(p, 1)_{\mathcal{T}_{h}} & =0,
\end{aligned}
$$

for all $(\boldsymbol{\tau}, \boldsymbol{v}, q, \boldsymbol{\mu}) \in \boldsymbol{W}_{h} \times \boldsymbol{V}_{h} \times P_{h} \times \boldsymbol{M}_{h}$. 
Applying the definition of the projections $\Pi$ and $(\Pi, \Pi)$, (2.4), and taking into account that $\boldsymbol{P}_{\partial}$ is nothing but the $L^{2}$-projection into $\boldsymbol{M}_{h}$, we easily get that

$$
\begin{aligned}
(\boldsymbol{\Pi} \boldsymbol{w}, \boldsymbol{\tau})_{\mathcal{T}_{h}}-(\boldsymbol{\Pi} \boldsymbol{u}, \nabla \times \boldsymbol{\tau})_{\mathcal{T}_{h}}-\left\langle\boldsymbol{P}_{\partial} \boldsymbol{u}, \boldsymbol{\tau} \times \boldsymbol{n}\right\rangle_{\partial \mathcal{T}_{h}} & =0 \\
(\boldsymbol{\Pi} \boldsymbol{w}, \nabla \times \boldsymbol{v})_{\mathcal{T}_{h}}-(\Pi p, \nabla \cdot \boldsymbol{v})_{\mathcal{T}_{h}} & \\
+\left\langle\boldsymbol{n} \times \boldsymbol{\Pi} \boldsymbol{w}+\Pi p \boldsymbol{n}+\tau_{n}\left(\boldsymbol{\Pi} \boldsymbol{u}-\boldsymbol{P}_{\partial} \boldsymbol{u}\right) \cdot \boldsymbol{n n}, \boldsymbol{v}\right\rangle_{\partial \mathcal{T}_{h}} & =(\boldsymbol{f}, \boldsymbol{v})_{\mathcal{T}_{h}} \\
& +\langle\boldsymbol{n} \times(\boldsymbol{\Pi} \boldsymbol{w}-\boldsymbol{w}), \boldsymbol{v}\rangle_{\partial \mathcal{T}_{h}}, \\
-(\boldsymbol{\Pi} \boldsymbol{u}, \nabla q)_{\mathcal{T}_{h}}+\left\langle\boldsymbol{P}_{\partial} \boldsymbol{u}, q \boldsymbol{n}\right\rangle_{\partial \mathcal{T}_{h}} & =0 \\
\left\langle\boldsymbol{n} \times \boldsymbol{\Pi} \boldsymbol{w}+\Pi p \boldsymbol{n}+\tau_{h}\left(\boldsymbol{\Pi} \boldsymbol{u}-\boldsymbol{P}_{\partial} \boldsymbol{u}\right) \cdot \boldsymbol{n n}, \boldsymbol{\mu}\right\rangle_{\partial \mathcal{T}_{h} \backslash \partial \Omega} & =\langle\boldsymbol{n} \times(\boldsymbol{\Pi} \boldsymbol{w}-\boldsymbol{w}), \boldsymbol{\mu}\rangle_{\partial \mathcal{T}_{h} \backslash \partial \Omega}, \\
\left\langle\boldsymbol{P}_{\partial} \boldsymbol{u}, \boldsymbol{\mu}\right\rangle_{\partial \Omega} & =\langle\boldsymbol{g}, \boldsymbol{\mu}\rangle_{\partial \Omega}, \\
(\Pi p, 1)_{\mathcal{T}_{h}} & =(\Pi p-p, 1)_{\mathcal{T}_{h}},
\end{aligned}
$$

and since, by definition of $\boldsymbol{S}_{\tau}, \tau_{n} \boldsymbol{\mu} \cdot \boldsymbol{n} \boldsymbol{n}=\boldsymbol{S}_{\tau} \boldsymbol{\mu}-\tau_{t} \boldsymbol{n} \times \boldsymbol{\mu} \times \boldsymbol{n}$, we obtain

$$
\begin{aligned}
& (\boldsymbol{\Pi} \boldsymbol{w}, \boldsymbol{\tau})_{\mathcal{T}_{h}}-(\boldsymbol{\Pi} \boldsymbol{u}, \nabla \times \boldsymbol{\tau})_{\mathcal{T}_{h}}-\left\langle\boldsymbol{P}_{\partial} \boldsymbol{u}, \boldsymbol{\tau} \times \boldsymbol{n}\right\rangle_{\partial \mathcal{T}_{h}}=0, \\
& (\boldsymbol{\Pi} \boldsymbol{w}, \nabla \times \boldsymbol{v})_{\mathcal{T}_{h}}-(\Pi p, \nabla \cdot \boldsymbol{v})_{\mathcal{T}_{h}} \\
& +\left\langle\boldsymbol{n} \times \boldsymbol{\Pi} \boldsymbol{w}+\Pi p \boldsymbol{n}+\boldsymbol{S}_{\tau}\left(\boldsymbol{\Pi} \boldsymbol{u}-\boldsymbol{P}_{\partial} \boldsymbol{u}\right), \boldsymbol{v}\right\rangle_{\partial \mathcal{T}_{h}}= \\
& (\boldsymbol{f}, \boldsymbol{v})_{\mathcal{T}_{h}}+\left\langle\boldsymbol{n} \times(\boldsymbol{\Pi} \boldsymbol{w}-\boldsymbol{w})+\tau_{t} \boldsymbol{n} \times\left(\boldsymbol{\Pi} \boldsymbol{u}-\boldsymbol{P}_{\partial} \boldsymbol{u}\right) \times \boldsymbol{n}, \boldsymbol{v}\right\rangle_{\partial \mathcal{T}_{h}}= \\
& (\boldsymbol{f}, \boldsymbol{v})_{\mathcal{T}_{h}}+\left\langle\boldsymbol{n} \times(\boldsymbol{\Pi} \boldsymbol{w}-\boldsymbol{w})+\boldsymbol{S}_{\tau}\left(\boldsymbol{\Pi} \boldsymbol{u}-\boldsymbol{P}_{\partial} \boldsymbol{u}\right), \boldsymbol{v}_{t}\right\rangle_{\partial \mathcal{T}_{h}}, \\
& -(\boldsymbol{\Pi} \boldsymbol{u}, \nabla q)_{\mathcal{T}_{h}}+\left\langle\boldsymbol{P}_{\partial} \boldsymbol{u}, q \boldsymbol{n}\right\rangle_{\partial \mathcal{T}_{h}}=0, \\
& \left\langle\boldsymbol{n} \times \boldsymbol{\Pi} \boldsymbol{w}+\Pi p \boldsymbol{n}+\boldsymbol{S}_{\tau}\left(\boldsymbol{\Pi} \boldsymbol{u}-\boldsymbol{P}_{\partial} \boldsymbol{u}\right), \boldsymbol{\mu}\right\rangle_{\partial \mathcal{T}_{h} \backslash \partial \Omega}= \\
& \left\langle\boldsymbol{n} \times(\boldsymbol{\Pi} \boldsymbol{w}-\boldsymbol{w})+\tau_{t} \boldsymbol{n} \times\left(\boldsymbol{\Pi} \boldsymbol{u}-\boldsymbol{P}_{\partial} \boldsymbol{u}\right) \times \boldsymbol{n}, \boldsymbol{\mu}\right\rangle_{\partial \mathcal{T}_{h} \backslash \partial \Omega}= \\
& \left\langle\boldsymbol{n} \times(\boldsymbol{\Pi} \boldsymbol{w}-\boldsymbol{w})+\boldsymbol{S}_{\tau}\left(\boldsymbol{\Pi} \boldsymbol{u}-\boldsymbol{P}_{\partial} \boldsymbol{u}\right), \boldsymbol{\mu}_{t}\right\rangle_{\partial \mathcal{T}_{h} \backslash \partial \Omega}, \\
& \left\langle\boldsymbol{P}_{\partial} \boldsymbol{u}, \boldsymbol{\mu}\right\rangle_{\partial \Omega}=\langle\boldsymbol{g}, \boldsymbol{\mu}\rangle_{\partial \Omega}, \\
& (\Pi p, 1)_{\mathcal{T}_{h}}=(\Pi p-p, 1)_{\mathcal{T}_{h}} .
\end{aligned}
$$

Finally, the error equations follow by subtracting the equations defining the HDG method, (2.2), from the above equations and applying the definitions of the projection of the errors. This completes the proof of Lemma 3.1 .

3.2. Estimate of the projection of the error in the vorticity. Define the seminorm

$$
\|\boldsymbol{\mu}\|_{\boldsymbol{S}_{\tau}}:=\left\langle\boldsymbol{S}_{\tau} \boldsymbol{\mu}, \boldsymbol{\mu}\right\rangle_{\partial \mathcal{T}_{h}}^{1 / 2}=\left[\left\langle\tau_{t} \boldsymbol{\mu}_{t}, \boldsymbol{\mu}_{t}\right\rangle_{\partial \mathcal{T}_{h}}+\left\langle\tau_{n} \boldsymbol{\mu} \cdot \boldsymbol{n}, \boldsymbol{\mu} \cdot \boldsymbol{n}\right\rangle_{\partial \mathcal{T}_{h}}\right]^{1 / 2}
$$

Lemma 3.2. We have

$$
\left\|\boldsymbol{\epsilon}^{\boldsymbol{w}}\right\|_{\Omega}^{2}+\left\|\boldsymbol{\epsilon}^{\boldsymbol{u}}-\boldsymbol{\epsilon}^{\widehat{\boldsymbol{u}}}\right\|_{\boldsymbol{S}_{\tau}}^{2}=\left\langle\boldsymbol{n} \times(\boldsymbol{\Pi} \boldsymbol{w}-\boldsymbol{w})+\tau_{t}\left(\boldsymbol{\Pi} \boldsymbol{u}-\boldsymbol{P}_{\partial} \boldsymbol{u}\right)_{t},\left(\boldsymbol{\epsilon}^{\boldsymbol{u}}-\boldsymbol{\epsilon}^{\widehat{\boldsymbol{u}}}\right)_{t}\right\rangle_{\partial \mathcal{T}_{h}} .
$$

Proof. We take $\boldsymbol{\tau}:=\boldsymbol{\epsilon}^{\boldsymbol{w}}, \boldsymbol{v}:=\boldsymbol{\epsilon}^{\boldsymbol{u}}$ and $q:=\epsilon^{p}$ in the first three equations of the error equation (3.1), $\boldsymbol{\mu}:=-\boldsymbol{\epsilon}^{\widehat{\boldsymbol{u}}}$ in (3.1d), and $\boldsymbol{\mu}:=-\left(\boldsymbol{n} \times \boldsymbol{\epsilon}^{\boldsymbol{w}}+\epsilon^{p} \boldsymbol{n}+\boldsymbol{S}_{\tau}\left(\boldsymbol{\epsilon}^{\boldsymbol{u}}-\boldsymbol{\epsilon}^{\widehat{\boldsymbol{u}}}\right)\right)$ in (3.1e). Adding the resulting equations, we obtain

$$
\begin{aligned}
\left(\boldsymbol{\epsilon}^{\boldsymbol{w}}, \boldsymbol{\epsilon}^{\boldsymbol{w}}\right)_{\mathcal{T}_{h}}+\Theta_{h} & =\left\langle\boldsymbol{n} \times(\boldsymbol{\Pi} \boldsymbol{w}-\boldsymbol{w})+\boldsymbol{S}_{\tau}\left(\Pi \boldsymbol{u}-\boldsymbol{P}_{\partial} \boldsymbol{u}\right),\left(\boldsymbol{\epsilon}^{\boldsymbol{u}}-\boldsymbol{\epsilon}^{\widehat{\boldsymbol{u}}}\right)_{t}\right\rangle_{\partial \mathcal{T}_{h}} \\
& =\left\langle\boldsymbol{n} \times(\boldsymbol{\Pi} \boldsymbol{w}-\boldsymbol{w})+\tau_{t}\left(\Pi \boldsymbol{u}-\boldsymbol{P}_{\partial} \boldsymbol{u}\right)_{t},\left(\boldsymbol{\epsilon}^{\boldsymbol{u}}-\boldsymbol{\epsilon}^{\widehat{\boldsymbol{u}}}\right)_{t}\right\rangle_{\partial \mathcal{T}_{h}},
\end{aligned}
$$


where

$$
\begin{aligned}
\Theta_{h}= & -\left(\boldsymbol{\epsilon}^{\boldsymbol{u}}, \nabla \times \boldsymbol{\epsilon}^{\boldsymbol{w}}\right)_{\mathcal{T}_{h}}-\left\langle\boldsymbol{\epsilon}^{\widehat{u}}, \boldsymbol{\epsilon}^{\boldsymbol{w}} \times \boldsymbol{n}\right\rangle_{\partial \mathcal{T}_{h}} \\
& +\left(\boldsymbol{\epsilon}^{\boldsymbol{w}}, \nabla \times \boldsymbol{\epsilon}^{\boldsymbol{u}}\right)_{\mathcal{T}_{h}}-\left(\epsilon^{p}, \nabla \cdot \boldsymbol{\epsilon}^{\boldsymbol{u}}\right)_{\mathcal{T}_{h}}+\left\langle\boldsymbol{n} \times \boldsymbol{\epsilon}^{\boldsymbol{w}}+\boldsymbol{\epsilon}^{p} \boldsymbol{n}+\boldsymbol{S}_{\tau}\left(\boldsymbol{\epsilon}^{\boldsymbol{u}}-\boldsymbol{\epsilon}^{\widehat{u}}\right), \boldsymbol{\epsilon}^{\boldsymbol{u}}\right\rangle_{\partial \mathcal{T}_{h}} \\
& -\left(\boldsymbol{\epsilon}^{\boldsymbol{u}}, \nabla \epsilon^{p}\right)_{\mathcal{T}_{h}}+\left\langle\boldsymbol{\epsilon}^{\widehat{u}}, \epsilon^{p} \boldsymbol{n}\right\rangle_{\partial \mathcal{T}_{h}} \\
& -\left\langle\boldsymbol{n} \times \boldsymbol{\epsilon}^{\boldsymbol{w}}+\boldsymbol{\epsilon}^{p} \boldsymbol{n}+\boldsymbol{S}_{\tau}\left(\boldsymbol{\epsilon}^{\boldsymbol{u}}-\boldsymbol{\epsilon}^{\widehat{\boldsymbol{u}}}\right), \boldsymbol{\epsilon}^{\widehat{u}}\right\rangle_{\partial \mathcal{T}_{h} \backslash \partial \Omega} \\
& -\left\langle\boldsymbol{n} \times \boldsymbol{\epsilon}^{\boldsymbol{w}}+\boldsymbol{\epsilon}^{p} \boldsymbol{n}+\boldsymbol{S}_{\tau}\left(\boldsymbol{\epsilon}^{\boldsymbol{u}}-\boldsymbol{\epsilon}^{\widehat{\boldsymbol{u}}}\right), \boldsymbol{\epsilon}^{\widehat{u}}\right\rangle_{\partial \Omega} .
\end{aligned}
$$

After simple rearrangements, we get that

$$
\begin{aligned}
\Theta_{h}= & -\left(\boldsymbol{\epsilon}^{\boldsymbol{u}}, \nabla \times \boldsymbol{\epsilon}^{\boldsymbol{w}}\right)_{\mathcal{T}_{h}}+\left\langle\boldsymbol{\epsilon}^{\boldsymbol{u}}, \boldsymbol{\epsilon}^{\boldsymbol{w}} \times \boldsymbol{n}\right\rangle_{\partial \mathcal{T}_{h}}+\left(\boldsymbol{\epsilon}^{\boldsymbol{w}}, \nabla \times \boldsymbol{\epsilon}^{\boldsymbol{u}}\right)_{\mathcal{T}_{h}} \\
& +\left(\epsilon^{p}, \nabla \cdot \boldsymbol{\epsilon}^{\boldsymbol{u}}\right)_{\mathcal{T}_{h}}-\left(\boldsymbol{\epsilon}^{\boldsymbol{u}}, \nabla \epsilon^{p}\right)_{\mathcal{T}_{h}}-\left\langle\boldsymbol{\epsilon}^{\boldsymbol{u}}, \epsilon^{p} \boldsymbol{n}\right\rangle_{\partial \mathcal{T}_{h}} \\
& +\left\langle\boldsymbol{S}_{\tau}\left(\boldsymbol{\epsilon}^{\boldsymbol{u}}-\boldsymbol{\epsilon}^{\widehat{\boldsymbol{u}}}\right), \boldsymbol{\epsilon}^{\boldsymbol{u}}-\boldsymbol{\epsilon}^{\widehat{u}}\right\rangle_{\partial \mathcal{T}_{h}} \\
= & \left\langle\boldsymbol{S}_{\tau}\left(\boldsymbol{\epsilon}^{\boldsymbol{u}}-\boldsymbol{\epsilon}^{\widehat{\boldsymbol{u}}}\right), \boldsymbol{\epsilon}^{\boldsymbol{u}}-\boldsymbol{\epsilon}^{\widehat{\boldsymbol{u}}}\right\rangle_{\partial \mathcal{T}_{h}} .
\end{aligned}
$$

This completes the proof.

The following estimate is a direct consequence of the above result.

Corollary 3.3. If $\tau_{t}>0$ on $\partial \mathcal{T}_{h}$, we have that

$$
\left\|\boldsymbol{\epsilon}^{\boldsymbol{w}}\right\|_{\Omega}^{2}+\left\|\boldsymbol{\epsilon}^{\boldsymbol{u}}-\boldsymbol{\epsilon}^{\widehat{\boldsymbol{u}}}\right\|_{\boldsymbol{S}_{\tau}}^{2} \leq\left\|\left(\boldsymbol{\Pi} \boldsymbol{w}-\boldsymbol{w}, \boldsymbol{\Pi} \boldsymbol{u}-\boldsymbol{P}_{\partial} \boldsymbol{u}\right)\right\|_{\tau_{t}, \partial \mathcal{T}_{h}}^{2} .
$$

3.3. Estimate of the projection of the error in the pressure. Next, we obtain an estimate for the projection of the error of the pressure. To do that, we use the well-known fact that, for any function $\zeta \in L_{2}(\Omega)$ such that $(\zeta, 1)_{\Omega}=0$, we have

$$
\|\zeta\|_{\Omega} \leq C \sup _{\mathbf{w} \in \boldsymbol{H}_{0}^{1}(\Omega) \backslash\{\mathbf{0}\}} \frac{(\zeta, \nabla \cdot \mathbf{w})_{\Omega}}{\|\mathbf{w}\|_{\boldsymbol{H}^{1}(\Omega)}},
$$

and take $\zeta=\epsilon^{p}-\overline{\epsilon^{p}}$. First, we obtain a suitable expression for $\left(\epsilon^{p}, \nabla \cdot \mathbf{w}\right)_{\Omega}$.

Lemma 3.4. Let $\boldsymbol{P}: \boldsymbol{H}^{1}\left(\mathcal{T}_{h}\right) \rightarrow \boldsymbol{V}_{h}$ be any projection such that $(\boldsymbol{P} \mathbf{w}-\mathbf{w}, \boldsymbol{v})_{T}=0$ for all $\boldsymbol{v} \in \boldsymbol{P}_{k-1}(T)$ for all $T \in \mathcal{T}_{h}$. Then,

$$
\begin{aligned}
\left(\epsilon^{p}, \nabla \cdot \mathbf{w}\right)_{\Omega}= & \left(\boldsymbol{\epsilon}^{\boldsymbol{w}}, \nabla \times \mathbf{w}\right)_{\mathcal{T}_{h}}+\left\langle\boldsymbol{S}_{\tau}\left(\boldsymbol{\epsilon}^{\boldsymbol{u}}-\boldsymbol{\epsilon}^{\widehat{u}}\right), \boldsymbol{P} \mathbf{w}-\boldsymbol{P}_{\partial} \mathbf{w}\right\rangle_{\partial \mathcal{T}_{h}} \\
& -\left\langle\boldsymbol{n} \times(\boldsymbol{\Pi} \boldsymbol{w}-\boldsymbol{w})+\boldsymbol{S}_{\tau}\left(\boldsymbol{\Pi} \boldsymbol{u}-\boldsymbol{P}_{\partial} \boldsymbol{u}\right),\left(\boldsymbol{P} \mathbf{w}-\boldsymbol{P}_{\partial} \mathbf{w}\right)_{t}\right\rangle_{\partial \mathcal{T}_{h}} .
\end{aligned}
$$

Proof. We have

$$
\left(\epsilon^{p}, \nabla \cdot \mathbf{w}\right)_{\Omega}=\left\langle\epsilon^{p}, \mathbf{w} \cdot \boldsymbol{n}\right\rangle_{\partial \mathcal{T}_{h}}-\left(\nabla \epsilon^{p}, \mathbf{w}\right)_{\mathcal{T}_{h}}=\left\langle\epsilon^{p}, \mathbf{w} \cdot \boldsymbol{n}\right\rangle_{\partial \mathcal{T}_{h}}-\left(\nabla \epsilon^{p}, \boldsymbol{P} \mathbf{w}\right)_{\mathcal{T}_{h}},
$$

since $\left.\nabla \epsilon^{p}\right|_{T} \in \boldsymbol{P}_{k-1}(T)$ for all $T \in \mathcal{T}_{h}$. By the error equation (3.1b) with $\boldsymbol{v}:=\boldsymbol{P} \mathbf{w}$, we get

$$
\begin{aligned}
\left(\epsilon^{p}, \nabla \cdot \mathbf{w}\right)_{\Omega}= & \left\langle\epsilon^{p}, \mathbf{w} \cdot \boldsymbol{n}\right\rangle_{\partial \mathcal{T}_{h}}+\left(\boldsymbol{\epsilon}^{\boldsymbol{w}}, \nabla \times \boldsymbol{P} \mathbf{w}\right)_{\mathcal{T}_{h}}+\left\langle\boldsymbol{n} \times \boldsymbol{\epsilon}^{\boldsymbol{w}}+\boldsymbol{S}_{\tau}\left(\boldsymbol{\epsilon}^{\boldsymbol{u}}-\boldsymbol{\epsilon}^{\widehat{u}}\right), \boldsymbol{P} \mathbf{w}\right\rangle_{\partial \mathcal{T}_{h}} \\
& -\left\langle\boldsymbol{n} \times(\boldsymbol{\Pi} \boldsymbol{w}-\boldsymbol{w})+\boldsymbol{S}_{\tau}\left(\boldsymbol{\Pi} \boldsymbol{u}-\boldsymbol{P}_{\partial} \boldsymbol{u}\right), \boldsymbol{P} \mathbf{w}_{t}\right\rangle_{\partial \mathcal{T}_{h}} .
\end{aligned}
$$

By the error equation (3.1d) with $\boldsymbol{\mu}:=\boldsymbol{P}_{\partial} \mathbf{w}$, and taking into account that $\boldsymbol{P}_{\partial} \mathbf{w}=\mathbf{0}$ on $\Omega$, we get

$$
\begin{aligned}
\left(\epsilon^{p}, \nabla \cdot \mathbf{w}\right)_{\Omega}= & \left(\boldsymbol{\epsilon}^{\boldsymbol{w}}, \nabla \times \boldsymbol{P} \mathbf{w}\right)_{\mathcal{T}_{h}}+\left\langle\boldsymbol{n} \times \boldsymbol{\epsilon}^{\boldsymbol{w}}+\boldsymbol{S}_{\tau}\left(\boldsymbol{\epsilon}^{\boldsymbol{u}}-\boldsymbol{\epsilon}^{\widehat{\boldsymbol{u}}}\right), \boldsymbol{P} \mathbf{w}-\boldsymbol{P}_{\partial} \mathbf{w}\right\rangle_{\partial \mathcal{T}_{h}} \\
& -\left\langle\boldsymbol{n} \times(\boldsymbol{\Pi} \boldsymbol{w}-\boldsymbol{w})+\boldsymbol{S}_{\tau}\left(\boldsymbol{\Pi} \boldsymbol{u}-\boldsymbol{P}_{\partial} \boldsymbol{u}\right),\left(\boldsymbol{P} \mathbf{w}-\boldsymbol{P}_{\partial} \mathbf{w}\right)_{t}\right\rangle_{\partial \mathcal{T}_{h}} .
\end{aligned}
$$


Integrating by parts the first term, and using the property of the auxiliary projection $\boldsymbol{P}$, we get

$$
\begin{aligned}
\left(\epsilon^{p}, \nabla \cdot \mathbf{w}\right)_{\Omega}= & \left(\nabla \times \boldsymbol{\epsilon}^{\boldsymbol{w}}, \mathbf{w}\right)_{\mathcal{T}_{h}}-\left\langle\boldsymbol{n} \times \boldsymbol{\epsilon}^{\boldsymbol{w}}, \boldsymbol{P}_{\partial} \mathbf{w}\right\rangle_{\partial \mathcal{T}_{h}}+\left\langle\boldsymbol{S}_{\tau}\left(\boldsymbol{\epsilon}^{\boldsymbol{u}}-\boldsymbol{\epsilon}^{\widehat{u}}\right), \boldsymbol{P} \mathbf{w}-\boldsymbol{P}_{\partial} \mathbf{w}\right\rangle_{\partial \mathcal{T}_{h}} \\
& -\left\langle\boldsymbol{n} \times(\boldsymbol{\Pi} \boldsymbol{w}-\boldsymbol{w})+\boldsymbol{S}_{\tau}\left(\boldsymbol{\Pi} \boldsymbol{u}-\boldsymbol{P}_{\partial} \boldsymbol{u}\right),\left(\boldsymbol{P} \mathbf{w}-\boldsymbol{P}_{\partial} \mathbf{w}\right)_{t}\right\rangle_{\partial \mathcal{T}_{h}},
\end{aligned}
$$

and the result follows after integrating by parts the first term of the right-hand side. This completes the proof.

Now, following [13, we introduce the operator $\overline{\boldsymbol{P}}: \boldsymbol{H}^{1}(T) \rightarrow \boldsymbol{P}_{k}(T)$ defined by

$$
\begin{aligned}
& (\overline{\boldsymbol{P}} \mathbf{w}-\mathbf{w}, \boldsymbol{v})_{T}=0 \quad \forall \boldsymbol{v} \in \boldsymbol{P}_{k-1}(T), \\
& \langle(\overline{\boldsymbol{P}} \mathbf{w}-\mathbf{w}) \cdot \boldsymbol{n}, \boldsymbol{v} \cdot \boldsymbol{n}\rangle_{\partial T}=0 \quad \forall \boldsymbol{v} \in \boldsymbol{P}_{k}(T)^{\perp},
\end{aligned}
$$

where $\boldsymbol{P}_{k}(T)^{\perp}$ is the space of polynomials in $\boldsymbol{P}_{k}(T)$ which are $L^{2}(K)$-orthogonal to all polynomials in $\boldsymbol{P}_{k-1}(T)$.

Lemma 3.5. We have

$$
\left\langle\left(\boldsymbol{\epsilon}^{\boldsymbol{u}}-\boldsymbol{\epsilon}^{\widehat{u}}\right) \cdot \boldsymbol{n},\left(\overline{\boldsymbol{P}} \mathbf{w}-\boldsymbol{P}_{\partial} \mathbf{w}\right) \cdot \boldsymbol{n}\right\rangle_{\partial T}=0 .
$$

Proof. Note that, by the error equations (3.1c),

$$
\left\langle\left(\boldsymbol{\epsilon}^{\boldsymbol{u}}-\boldsymbol{\epsilon}^{\widehat{\boldsymbol{u}}}\right) \cdot \boldsymbol{n}, q\right\rangle_{\partial T}=0
$$

for all $q \in P_{k}(T)$ which is $L^{2}(T)$-orthogonal to polynomials in $P_{k-1}(T)$. By Lemma 5.1 in [13], this implies that there exists a function $\boldsymbol{v} \in \boldsymbol{P}_{k}(T)^{\perp}$ such that $\left(\boldsymbol{\epsilon}^{\boldsymbol{u}}-\right.$ $\left.\epsilon^{\widehat{u}}\right) \cdot \boldsymbol{n}=\boldsymbol{v} \cdot \boldsymbol{n}$ on $\partial K$. As a consequence, the result follows by the second equation defining the projection $\overline{\boldsymbol{P}},(3.3 \mathrm{~b})$. This completes the proof.

Corollary 3.6. We have that

$$
\left\|\epsilon^{p}-\overline{(\Pi p-p)}\right\|_{\Omega} \leq C C_{\tau_{t}}\left\|\left(\boldsymbol{\Pi} \boldsymbol{w}-\boldsymbol{w}, \boldsymbol{\Pi} \boldsymbol{u}-\boldsymbol{P}_{\partial} \boldsymbol{u}\right)\right\|_{\tau_{t}, \partial \mathcal{T}_{h}},
$$

where $C_{\tau_{t}}$ is defined in Theorem 2.3.

Proof. By Lemma 3.4 and Lemma 3.5 we get

$$
\begin{aligned}
\left(\epsilon^{p}, \nabla \cdot \mathbf{w}\right)_{\Omega}= & \left(\boldsymbol{\epsilon}^{\boldsymbol{w}}, \nabla \times \mathbf{w}\right)_{\mathcal{T}_{h}}+\left\langle\boldsymbol{S}_{\tau}\left(\boldsymbol{\epsilon}^{\boldsymbol{u}}-\boldsymbol{\epsilon}^{\widehat{u}}\right), \overline{\boldsymbol{P}} \mathbf{w}-\boldsymbol{P}_{\partial} \mathbf{w}\right\rangle_{\partial \mathcal{T}_{h}} \\
& -\left\langle\boldsymbol{n} \times(\boldsymbol{\Pi} \boldsymbol{w}-\boldsymbol{w})+\boldsymbol{S}_{\tau}\left(\boldsymbol{\Pi} \boldsymbol{u}-\boldsymbol{P}_{\partial} \boldsymbol{u}\right),\left(\overline{\boldsymbol{P}} \mathbf{w}-\boldsymbol{P}_{\partial} \mathbf{w}\right)_{t}\right\rangle_{\partial \mathcal{T}_{h}} \\
= & \left(\boldsymbol{\epsilon}^{\boldsymbol{w}}, \nabla \times \mathbf{w}\right)_{\mathcal{T}_{h}}+\left\langle\boldsymbol{S}_{\tau}\left(\boldsymbol{\epsilon}^{\boldsymbol{u}}-\boldsymbol{\epsilon}^{\widehat{u}}\right),\left(\overline{\boldsymbol{P}} \mathbf{w}-\boldsymbol{P}_{\partial} \mathbf{w}\right)_{t}\right\rangle_{\partial \mathcal{T}_{h}} \\
& -\left\langle\boldsymbol{n} \times(\boldsymbol{\Pi} \boldsymbol{w}-\boldsymbol{w})+\boldsymbol{S}_{\tau}\left(\boldsymbol{\Pi} \boldsymbol{u}-\boldsymbol{P}_{\partial} \boldsymbol{u}\right),\left(\overline{\boldsymbol{P}} \mathbf{w}-\boldsymbol{P}_{\partial} \mathbf{w}\right)_{t}\right\rangle_{\partial \mathcal{T}_{h}} .
\end{aligned}
$$

This implies that

$$
\begin{array}{r}
\left(\epsilon^{p}, \nabla \cdot \mathbf{w}\right)_{\Omega} \leq\left\|\boldsymbol{\epsilon}^{\boldsymbol{w}}\right\|_{\mathcal{T}_{h}}\|\nabla \times \mathbf{w}\|_{\mathcal{T}_{h}}+\left\|\boldsymbol{\epsilon}^{\boldsymbol{u}}-\boldsymbol{\epsilon}^{\widehat{\boldsymbol{u}}}\right\|_{\boldsymbol{S}_{\tau}}\left\|\left(\overline{\boldsymbol{P}} \mathbf{w}-\boldsymbol{P}_{\partial} \mathbf{w}\right)_{t}\right\|_{\boldsymbol{S}_{\tau}} \\
\left\|\left(\boldsymbol{\Pi} \boldsymbol{w}-\boldsymbol{w}, \boldsymbol{\Pi} \boldsymbol{u}-\boldsymbol{P}_{\partial} \boldsymbol{u}\right)\right\|_{\tau_{t}, \partial \mathcal{T}_{h}}\left\|\left(\overline{\boldsymbol{P}} \mathbf{w}-\boldsymbol{P}_{\partial} \mathbf{w}\right)_{t}\right\|_{\boldsymbol{S}_{\tau}} .
\end{array}
$$

Finally, by Corollary 3.3 .

$$
\left(\epsilon^{p}, \nabla \cdot \mathbf{w}\right)_{\Omega} \leq\left\|\left(\boldsymbol{\Pi} \boldsymbol{w}-\boldsymbol{w}, \boldsymbol{\Pi} \boldsymbol{u}-\boldsymbol{P}_{\partial} \boldsymbol{u}\right)\right\|_{\tau_{t}, \partial \mathcal{T}_{h}} \Theta_{h}
$$

where

$$
\Theta_{h}=\|\nabla \times \mathbf{w}\|_{\mathcal{T}_{h}}+2\left\|\left(\overline{\boldsymbol{P}} \mathbf{w}-\boldsymbol{P}_{\partial} \mathbf{w}\right)_{t}\right\|_{\boldsymbol{S}_{\tau}} \leq C C_{\tau_{t}}\|\mathbf{w}\|_{\boldsymbol{H}^{1}},
$$

by Proposition 4.4 in $[13$. 
The result now follows from the fact that

$$
\left\|\epsilon^{p}-\overline{\epsilon^{p}}\right\|_{\Omega} \leq C \sup _{\mathbf{w} \in \boldsymbol{H}_{0}^{1}(\Omega) \backslash\{\mathbf{0}\}} \frac{\left(\epsilon^{p}, \nabla \cdot \mathbf{w}\right)_{\Omega}}{\|\mathbf{w}\|_{\boldsymbol{H}^{1}(\Omega)}},
$$

and that $\overline{\epsilon^{p}}=\overline{\Pi p-p}$, by the last error equation (3.1f). This completes the proof.

3.4. Estimate of the projection of the error in the velocity. In order to proceed with the estimate of the velocity by using duality argument, we first present several properties of the projection $(\boldsymbol{\Pi}, \boldsymbol{\Pi}, \Pi)$.

Lemma 3.7. Assume that $\boldsymbol{\psi}, \phi, \phi$ are arbitrary functions in $\boldsymbol{H}^{1}\left(\mathcal{T}_{h}\right)$, $\boldsymbol{H}^{1}\left(\mathcal{T}_{h}\right), H^{1}\left(\mathcal{T}_{h}\right)$. Then we have

$$
\begin{aligned}
(\boldsymbol{w}, \nabla \times \boldsymbol{\psi})_{\mathcal{T}_{h}} & =(\boldsymbol{w}, \nabla \times(\boldsymbol{\Pi} \boldsymbol{\psi}))_{\mathcal{T}_{h}}-\langle\boldsymbol{w},(\boldsymbol{\psi}-\boldsymbol{\Pi} \boldsymbol{\psi}) \times \boldsymbol{n}\rangle_{\partial \mathcal{T}_{h}}, \\
(q, \nabla \cdot \boldsymbol{\phi})_{\mathcal{T}_{h}} & =\langle q \boldsymbol{n}, \boldsymbol{\phi}\rangle_{\partial \mathcal{T}_{h}}+(\nabla q, \boldsymbol{\Pi} \boldsymbol{\phi})_{\mathcal{T}_{h}}, \\
(\boldsymbol{v}, \nabla \times \boldsymbol{\phi})_{\mathcal{T}_{h}} & =(\nabla \times \boldsymbol{v}, \boldsymbol{\Pi} \phi)_{\mathcal{T}_{h}}+\langle\boldsymbol{v} \times \boldsymbol{n}, \boldsymbol{\phi}\rangle_{\partial \mathcal{T}_{h}}, \\
(\boldsymbol{v}, \nabla \phi)_{\mathcal{T}_{h}} & =(\boldsymbol{v}, \nabla(\Pi \phi))_{\mathcal{T}_{h}}+\langle\boldsymbol{v},(\phi-\Pi \phi) \boldsymbol{n}\rangle_{\partial \mathcal{T}_{h}},
\end{aligned}
$$

for all $(\boldsymbol{w}, \boldsymbol{v}, q) \in \boldsymbol{W}_{h} \times \boldsymbol{V}_{h} \times P_{h}$.

Proof. We will prove (3.4a) first. By integration by parts and 2.4a), we have

$$
\begin{aligned}
(\boldsymbol{w}, \nabla \times \boldsymbol{\psi})_{\mathcal{T}_{h}} & =(\nabla \times \boldsymbol{w}, \boldsymbol{\psi})_{\mathcal{T}_{h}}-\langle\boldsymbol{w}, \boldsymbol{\psi} \times \boldsymbol{n}\rangle_{\partial \mathcal{T}_{h}} \\
& =(\nabla \times \boldsymbol{w}, \boldsymbol{\Pi} \boldsymbol{\psi})_{\mathcal{T}_{h}}-\langle\boldsymbol{w}, \boldsymbol{\psi} \times \boldsymbol{n}\rangle_{\partial \mathcal{T}_{h}} .
\end{aligned}
$$

Integrating by parts again, we get

$$
(\boldsymbol{w}, \nabla \times \boldsymbol{\psi})_{\mathcal{T}_{h}}=(\boldsymbol{w}, \nabla \times(\boldsymbol{\Pi} \boldsymbol{\psi}))_{\mathcal{T}_{h}}-\langle\boldsymbol{w},(\boldsymbol{\psi}-\boldsymbol{\Pi} \boldsymbol{\psi}) \times \boldsymbol{n}\rangle_{\partial \mathcal{T}_{h}} .
$$

This completes the proof for the first identity. The rest of the identities in (3.4) can be proven similarly.

We now use a duality argument to derive the estimate in the velocity.

Proposition 3.8. We have

$$
\begin{aligned}
\left(\boldsymbol{\epsilon}^{\boldsymbol{u}}, \boldsymbol{\theta}\right)_{\mathcal{T}_{h}}= & \left\langle\left(\boldsymbol{\epsilon}^{\boldsymbol{u}}-\boldsymbol{\epsilon}^{\widehat{\boldsymbol{u}}}\right)_{t},(\boldsymbol{\psi}-\boldsymbol{\Pi} \boldsymbol{\psi}) \times \boldsymbol{n}+\tau_{t}\left(\boldsymbol{P}_{\partial} \boldsymbol{\phi}-\boldsymbol{\Pi} \boldsymbol{\phi}\right)_{t}\right\rangle_{\partial \mathcal{T}_{h}} \\
& -\left\langle\boldsymbol{n} \times(\boldsymbol{\Pi} \boldsymbol{w}-\boldsymbol{w})+\tau_{t}\left(\boldsymbol{\Pi} \boldsymbol{u}-\boldsymbol{P}_{\partial} \boldsymbol{u}\right)_{t},\left(\boldsymbol{P}_{\partial} \boldsymbol{\phi}-\boldsymbol{\Pi} \boldsymbol{\phi}\right)_{t}\right\rangle_{\partial \mathcal{T}_{h}},
\end{aligned}
$$

where $\boldsymbol{\theta}, \boldsymbol{\phi}$ and $\boldsymbol{\psi}$ are the functions in the dual problem (2.5).

Proof. By the equations defining the dual problem, (2.5a)-(2.5c), we have

$$
\begin{aligned}
\left(\boldsymbol{\epsilon}^{\boldsymbol{u}}, \boldsymbol{\theta}\right)_{\mathcal{T}_{h}} & =\left(\boldsymbol{\epsilon}^{\boldsymbol{w}}, \boldsymbol{\psi}+\nabla \times \boldsymbol{\phi}\right)_{\mathcal{T}_{h}}-\left(\boldsymbol{\epsilon}^{\boldsymbol{u}}, \nabla \times \boldsymbol{\psi}+\nabla \phi\right)_{\mathcal{T}_{h}}-\left(\epsilon^{p}, \nabla \cdot \boldsymbol{\phi}\right)_{\mathcal{T}_{h}} \\
& =\left(\boldsymbol{\epsilon}^{\boldsymbol{w}}, \boldsymbol{\psi}\right)_{\mathcal{T}_{h}}-\left(\boldsymbol{\epsilon}^{\boldsymbol{u}}, \nabla \times \boldsymbol{\psi}\right)_{\mathcal{T}_{h}}+\left(\boldsymbol{\epsilon}^{\boldsymbol{w}}, \nabla \times \boldsymbol{\phi}\right)_{\mathcal{T}_{h}}-\left(\epsilon^{p}, \nabla \cdot \boldsymbol{\phi}\right)_{\mathcal{T}_{h}}-\left(\boldsymbol{\epsilon}^{\boldsymbol{u}}, \nabla \phi\right)_{\mathcal{T}_{h}} .
\end{aligned}
$$

Applying the orthogonality property (2.4a) to the first term of the right-hand side and the properties (3.4a)-(3.4d) to the last four terms, we obtain

$$
\begin{aligned}
\left(\boldsymbol{\epsilon}^{\boldsymbol{u}}, \boldsymbol{\theta}\right)_{\mathcal{T}_{h}}= & \left(\boldsymbol{\epsilon}^{\boldsymbol{w}}, \boldsymbol{\Pi} \boldsymbol{\psi}\right)_{\mathcal{T}_{h}}-\left(\boldsymbol{\epsilon}^{\boldsymbol{u}}, \nabla \times(\boldsymbol{\Pi} \psi)\right)_{\mathcal{T}_{h}}+\left\langle\boldsymbol{\epsilon}^{\boldsymbol{u}},(\boldsymbol{\psi}-\boldsymbol{\Pi} \boldsymbol{\psi}) \times \boldsymbol{n}\right\rangle_{\partial \mathcal{T}_{h}} \\
& +\left(\nabla \times \boldsymbol{\epsilon}^{\boldsymbol{w}}, \boldsymbol{\Pi} \phi\right)_{\mathcal{T}_{h}}+\left(\nabla \epsilon^{p}, \boldsymbol{\Pi} \phi\right)_{\mathcal{T}_{h}} \\
& +\left\langle\boldsymbol{\epsilon}^{\boldsymbol{w}} \times \boldsymbol{n}-\epsilon^{p} \boldsymbol{n}, \boldsymbol{\phi}\right\rangle_{\partial \mathcal{T}_{h}} \\
& -\left(\boldsymbol{\epsilon}^{\boldsymbol{u}}, \nabla(\Pi \phi)\right)_{\mathcal{T}_{h}}-\left\langle\boldsymbol{\epsilon}^{\boldsymbol{u}},(\phi-\Pi \phi) \boldsymbol{n}\right\rangle_{\partial \mathcal{T}_{h}} .
\end{aligned}
$$


Now, by using the first four error equations (3.1) we take $\tau:=\Pi \psi, v:=\boldsymbol{\Pi} \phi$, $q:=\Pi \phi$, and $\boldsymbol{\mu}:=\boldsymbol{P}_{\partial} \phi$, respectively, we get

$$
\begin{aligned}
\left(\boldsymbol{\epsilon}^{\boldsymbol{u}}, \boldsymbol{\theta}\right)= & \left\langle\boldsymbol{\epsilon}^{\widehat{\boldsymbol{u}}}, \boldsymbol{\Pi} \boldsymbol{\psi} \times \boldsymbol{n}\right\rangle_{\partial \mathcal{T}_{h}}+\left\langle\boldsymbol{\epsilon}^{\boldsymbol{u}},(\boldsymbol{\psi}-\boldsymbol{\Pi} \boldsymbol{\psi}) \times \boldsymbol{n}\right\rangle_{\partial \mathcal{T}_{h}} \\
& -\left\langle\boldsymbol{S}_{\tau}\left(\boldsymbol{\epsilon}^{\boldsymbol{u}}-\boldsymbol{\epsilon}^{\widehat{u}}\right), \boldsymbol{\Pi} \phi\right\rangle_{\partial \mathcal{T}_{h}}+\left\langle\boldsymbol{n} \times(\boldsymbol{\Pi} \boldsymbol{w}-\boldsymbol{w})+\boldsymbol{S}_{\tau}\left(\boldsymbol{\Pi} \boldsymbol{u}-\boldsymbol{P}_{\partial} \boldsymbol{u}\right),(\boldsymbol{\Pi} \phi)_{t}\right\rangle_{\partial \mathcal{T}_{h}} \\
& +\left\langle\boldsymbol{S}_{\tau}\left(\boldsymbol{\epsilon}^{\boldsymbol{u}}-\boldsymbol{\epsilon}^{\widehat{u}}\right), \boldsymbol{P}_{\partial} \boldsymbol{\phi}\right\rangle_{\partial \mathcal{T}_{h}}-\left\langle\boldsymbol{n} \times(\boldsymbol{\Pi} \boldsymbol{w}-\boldsymbol{w})+\boldsymbol{S}_{\tau}\left(\boldsymbol{\Pi} \boldsymbol{u}-\boldsymbol{P}_{\partial} \boldsymbol{u}\right),\left(\boldsymbol{P}_{\partial} \phi\right)_{t}\right\rangle_{\partial \mathcal{T}_{h}} \\
& +\left\langle\boldsymbol{\epsilon}^{\widehat{u}},(\Pi \phi) \boldsymbol{n}\right\rangle_{\partial \mathcal{T}_{h}}+\left\langle\boldsymbol{\epsilon}^{\boldsymbol{u}},(\phi-\Pi \phi) \boldsymbol{n}\right\rangle_{\partial \mathcal{T}_{h}} \\
= & \left\langle\boldsymbol{\epsilon}^{\widehat{\boldsymbol{u}}}, \boldsymbol{\psi} \times \boldsymbol{n}\right\rangle_{\partial \mathcal{T}_{h}}+\left\langle\boldsymbol{\epsilon}^{\boldsymbol{u}}-\boldsymbol{\epsilon}^{\widehat{u}},(\boldsymbol{\psi}-\boldsymbol{\Pi} \boldsymbol{\psi}) \times \boldsymbol{n}\right\rangle_{\partial \mathcal{T}_{h}} \\
& +\left\langle\boldsymbol{n} \times(\boldsymbol{\Pi} \boldsymbol{w}-\boldsymbol{w})+\boldsymbol{S}_{\tau}\left(\boldsymbol{\Pi} \boldsymbol{u}-\boldsymbol{P}_{\partial} \boldsymbol{u}\right),\left(\boldsymbol{\Pi} \boldsymbol{\phi}-\boldsymbol{P}_{\partial} \phi\right)_{t}\right\rangle_{\partial \mathcal{T}_{h}} \\
& +\left\langle\boldsymbol{S}_{\tau}\left(\boldsymbol{\epsilon}^{\boldsymbol{u}}-\boldsymbol{\epsilon}^{\widehat{u}}\right), \boldsymbol{P}_{\partial} \boldsymbol{\phi}-\boldsymbol{\Pi} \boldsymbol{\phi}\right\rangle_{\partial \mathcal{T}_{h}} \\
& +\left\langle\boldsymbol{\epsilon}^{\widehat{u}}, \phi \boldsymbol{n}\right\rangle_{\partial \mathcal{T}_{h}}+\left\langle\boldsymbol{\epsilon}^{\boldsymbol{u}}-\boldsymbol{\epsilon}^{\widehat{u}},(\phi-\Pi \phi) \boldsymbol{n}\right\rangle_{\partial \mathcal{T}_{h}} \\
= & \left\langle\boldsymbol{\epsilon}^{\widehat{\boldsymbol{u}}}, \boldsymbol{\psi} \times \boldsymbol{n}\right\rangle_{\partial \mathcal{T}_{h}}+\left\langle\boldsymbol{\epsilon}^{\boldsymbol{u}}-\boldsymbol{\epsilon}^{\widehat{u}},(\boldsymbol{\psi}-\boldsymbol{\Pi} \boldsymbol{\psi}) \times \boldsymbol{n}+\tau_{t}\left(\boldsymbol{P}_{\partial} \boldsymbol{\phi}-\boldsymbol{\Pi} \phi\right)_{t}\right\rangle_{\partial \mathcal{T}_{h}} \\
& +\left\langle\boldsymbol{n} \times(\boldsymbol{\Pi} \boldsymbol{w}-\boldsymbol{w})+\boldsymbol{S}_{\tau}\left(\boldsymbol{\Pi} \boldsymbol{u}-\boldsymbol{P}_{\partial} \boldsymbol{u}\right),\left(\boldsymbol{\Pi} \boldsymbol{\phi}-\boldsymbol{P}_{\partial} \boldsymbol{\phi}\right)_{t}\right\rangle_{\partial \mathcal{T}_{h}} \\
& +\left\langle\boldsymbol{\epsilon}^{\widehat{u}}, \phi \boldsymbol{n}\right\rangle_{\partial \mathcal{T}_{h}}+\left\langle\left(\boldsymbol{\epsilon}^{\boldsymbol{u}}-\boldsymbol{\epsilon}^{\widehat{u}}\right) \cdot \boldsymbol{n},(\phi-\Pi \phi)+\tau_{n}(\boldsymbol{\phi}-\boldsymbol{\Pi} \boldsymbol{\phi}) \cdot \boldsymbol{n}\right\rangle_{\partial \mathcal{T}_{h} .}
\end{aligned}
$$

This implies that

$$
\begin{aligned}
\left(\boldsymbol{\epsilon}^{\boldsymbol{u}}, \boldsymbol{\theta}\right)_{\mathcal{T}_{h}}= & \left\langle\left(\boldsymbol{\epsilon}^{\boldsymbol{u}}-\boldsymbol{\epsilon}^{\widehat{\boldsymbol{u}}}\right)_{t},(\boldsymbol{\psi}-\boldsymbol{\Pi} \boldsymbol{\psi}) \times \boldsymbol{n}+\tau_{t}(\boldsymbol{\phi}-\boldsymbol{\Pi} \boldsymbol{\phi})_{t}\right\rangle_{\partial \mathcal{T}_{h}} \\
& +\left\langle(\boldsymbol{\Pi} \boldsymbol{w}-\boldsymbol{w}) \times \boldsymbol{n}-\boldsymbol{S}_{\tau}\left(\boldsymbol{\Pi} \boldsymbol{u}-\boldsymbol{P}_{\partial} \boldsymbol{u}\right),\left(\boldsymbol{P}_{\partial} \boldsymbol{\phi}-\boldsymbol{\Pi}\right)_{t}\right\rangle_{\partial \mathcal{T}_{h}}+T_{1}+T_{2},
\end{aligned}
$$

where

$$
\begin{aligned}
& T_{1}=\left\langle\boldsymbol{\epsilon}^{\widehat{u}}, \boldsymbol{\psi} \times \boldsymbol{n}+\phi \boldsymbol{n}\right\rangle_{\partial \mathcal{T}_{h}}, \\
& T_{2}=\left\langle\left(\boldsymbol{\epsilon}^{\boldsymbol{u}}-\boldsymbol{\epsilon}^{\widehat{\boldsymbol{u}}}\right) \cdot \boldsymbol{n},(\phi-\Pi \phi)+\tau_{n}(\boldsymbol{\phi}-\boldsymbol{\Pi} \phi) \cdot \boldsymbol{n}\right\rangle_{\partial \mathcal{T}_{h}} .
\end{aligned}
$$

But $T_{1}=\left\langle\boldsymbol{\epsilon}^{\widehat{u}}, \boldsymbol{\psi} \times \boldsymbol{n}+\phi \boldsymbol{n}\right\rangle_{\partial \Omega}$ since $\boldsymbol{\epsilon}^{\widehat{u}}$ is a single-valued function on $\partial \mathcal{T}_{h}$. Moreover, $\left.\epsilon^{\widehat{u}}\right|_{\partial \Omega}=0$ by the error equation (3.1e), and we conclude that $T_{1}=0$.

We also have that $T_{2}=0$, by the orthogonality property of the auxiliary projection (2.4d). This completes the proof.

Corollary 3.9. Assume that the elliptic regularity inequality (2.6) holds. Then we have

$$
\left\|\boldsymbol{\epsilon}^{\boldsymbol{u}}\right\|_{\mathcal{T}_{h}} \leq C H_{\tau}\left\|\left(\boldsymbol{\Pi} \boldsymbol{w}-\boldsymbol{w}, \boldsymbol{\Pi} \boldsymbol{u}-\boldsymbol{P}_{\partial} \boldsymbol{u}\right)\right\|_{\tau_{t}, \partial \mathcal{T}_{h}},
$$

where $H_{\tau}$ is defined in Theorem 2.3 .

Proof. It directly follows from by Corollary 3.3 and Proposition 3.8 that

$$
\begin{aligned}
\left(\boldsymbol{\epsilon}^{\boldsymbol{u}}, \boldsymbol{\theta}\right)_{\mathcal{T}_{h} \leq} \leq & \left\|\boldsymbol{\epsilon}^{\boldsymbol{u}}-\boldsymbol{\epsilon}^{\widehat{\boldsymbol{u}}}\right\|_{\boldsymbol{S}_{\tau}\left\|\left(\boldsymbol{\psi}-\boldsymbol{\Pi} \boldsymbol{\psi}, \boldsymbol{P}_{\partial} \boldsymbol{\phi}-\boldsymbol{\Pi} \boldsymbol{\phi}\right)\right\|_{\tau_{t}, \partial \mathcal{T}_{h}}} \\
& +\left\|\left(\boldsymbol{\Pi} \boldsymbol{w}-\boldsymbol{w}, \boldsymbol{\Pi} \boldsymbol{u}-\boldsymbol{P}_{\partial} \boldsymbol{u}\right)\right\|_{\tau_{t}, \partial \mathcal{T}_{h}}\left\|\left(\boldsymbol{P}_{\partial} \boldsymbol{\phi}-\boldsymbol{\Pi} \boldsymbol{\phi}\right)_{t}\right\|_{\boldsymbol{S}_{\tau}} \\
\leq & \left.2 \| \boldsymbol{\psi}-\boldsymbol{\Pi} \boldsymbol{\psi}, \boldsymbol{P}_{\partial} \boldsymbol{\phi}-\boldsymbol{\Pi} \boldsymbol{\phi}\right)\left\|_{\tau_{t}, \partial \mathcal{T}_{h}}\right\|\left(\boldsymbol{\Pi} \boldsymbol{w}-\boldsymbol{w}, \boldsymbol{\Pi} \boldsymbol{u}-\boldsymbol{P}_{\partial} \boldsymbol{u}\right) \|_{\tau_{t}, \partial \mathcal{T}_{h}} .
\end{aligned}
$$

As a consequence, we get

$$
\left\|\boldsymbol{\epsilon}^{\boldsymbol{u}}\right\|_{\mathcal{T}_{h}} \leq D_{\tau_{t}}\left\|\left(\boldsymbol{\Pi} \boldsymbol{w}-\boldsymbol{w}, \boldsymbol{\Pi} \boldsymbol{u}-\boldsymbol{P}_{\partial} \boldsymbol{u}\right)\right\|_{\tau_{t}, \partial \mathcal{T}_{h}}
$$

where

$$
D_{\tau_{t}}:=2 \sup _{\boldsymbol{\theta} \in L^{2}(\Omega) \backslash\{0\}} \frac{\left\|\left(\boldsymbol{\psi}-\boldsymbol{\Pi} \boldsymbol{\psi}, \boldsymbol{P}_{\partial} \boldsymbol{\phi}-\boldsymbol{\Pi} \boldsymbol{\phi}\right)\right\|_{\tau_{t}, \partial \mathcal{T}_{h}}}{\|\boldsymbol{\theta}\|_{\Omega}} .
$$


But, by Proposition 2.2

$$
\begin{aligned}
\|(\boldsymbol{\Pi} \boldsymbol{\psi}-\boldsymbol{\psi}, \boldsymbol{\Pi} \boldsymbol{\phi}-\boldsymbol{\phi})\|_{\tau_{t}, \partial \mathcal{T}_{h}} \leq & C \max _{T \in \mathcal{T}_{h}} h_{T}^{1 / 2}\left\|\tau_{t}^{-1}\right\|_{L^{\infty}(\partial T)}^{1 / 2} E(\boldsymbol{\Pi} \boldsymbol{\psi}, \boldsymbol{\psi}) \\
& +C \max _{T \in \mathcal{T}_{h}} h_{T}^{1 / 2}\left\|\tau_{t}\right\|_{L^{\infty}(\partial T)}^{1 / 2} E(\boldsymbol{\Pi} \boldsymbol{\phi}, \boldsymbol{\phi}),
\end{aligned}
$$

and by the approximation properties of the projections,

$$
\begin{aligned}
& \|(\boldsymbol{\Pi} \boldsymbol{\psi}-\boldsymbol{\psi}, \boldsymbol{\Pi} \boldsymbol{\phi}-\boldsymbol{\phi})\|_{\tau_{t}, \partial \mathcal{T}_{h}} \leq C \max _{T \in \mathcal{T}_{h}} h_{T}^{1 / 2}\left\|\tau_{t}^{-1}\right\|_{L^{\infty}(\partial T)}^{1 / 2}\|\boldsymbol{\psi}\|_{H^{1}(\Omega)} \\
& \quad+C \max _{T \in \mathcal{T}_{h}} h_{T}^{1 / 2}\left\|\tau_{t}\right\|_{L^{\infty}(\partial T)}^{1 / 2}\left(\max _{T \in \mathcal{T}_{h}} h_{T}^{\min \{1, k\}}\|\phi\|_{H^{2}(\Omega)}\right. \\
& \left.\quad+\max _{T \in \mathcal{T}_{h}}\left\|\left(\tau_{n}^{*}\right)^{-1}\right\|_{L^{\infty}(\partial T)}\|\phi\|_{H^{1}(\Omega)}\right) .
\end{aligned}
$$

Finally, by using the elliptic regularity inequality (2.6) and the definition of the constant $H_{\tau}$, we get that

$$
\|(\boldsymbol{\Pi} \boldsymbol{\psi}-\boldsymbol{\psi}, \boldsymbol{\Pi} \boldsymbol{\phi}-\boldsymbol{\phi})\|_{\tau_{t}, \partial \mathcal{T}_{h}} \leq C H_{\tau}\|\boldsymbol{\theta}\|_{\Omega} .
$$

This completes the proof.

\section{Concluding Remarks}

Note that all the estimates of the projection of the errors are independent of the stabilization function $\tau_{n}$. Note also that the approximation errors of the auxiliary projection do hold in the limit as $\tau_{n}$ goes to infinity. This suggests that we should be able to take the limit and obtain $\boldsymbol{H}$ (div)-conforming HDG methods based on a vorticity-velocity-pressure formulation just as was done for HDG methods based on velocity gradient-velocity-pressure formulation; see [13. This constitutes the subject of ongoing research.

\section{Appendix A. Proof of the approximation result of Proposition 2.2}

In this section, we prove Proposition 2.2. To do that, we need to show how to estimate the error in the border on an element $T,\|Z-\zeta\|_{\partial T}$, in terms of the error inside the element, $\|Z-\zeta\|_{T}$.

Lemma A.1. Let $\zeta$ be any element of $H^{1}(T)$ and let $Z, S$ be two arbitrary elements of $P_{k}(T)$. Then

$$
\|Z-\zeta\|_{\partial T} \leq C h_{T}^{-1 / 2}\left(\|Z-\zeta\|_{T}+\|S-\zeta\|_{T}\right)+C h_{T}^{1 / 2}\|\nabla(S-\zeta)\|_{T},
$$

where the constant $C$ depends only on the shape-regularity constant of the simplex $T$ and on the polynomial degree $k$.

Proof. We have

$$
\|Z-\zeta\|_{\partial T} \leq\|Z-S\|_{\partial T}+\|S-\zeta\|_{\partial T} \leq C h_{T}^{-1 / 2}\|Z-S\|_{T}+\|S-\zeta\|_{\partial T},
$$

by a standard inverse inequality. Then, by the trace inequality,

$$
\|Z-\zeta\|_{\partial T} \leq C h_{T}^{-1 / 2}\left(\|Z-S\|_{T}+\|S-\zeta\|_{T}\right)+C h_{T}^{1 / 2}\|\nabla(S-\zeta)\|_{T},
$$

and the result follows by the triangle inequality.

We are now ready to prove Proposition 2.2 . 
Proof. Since, by definition of the seminorm $\|\cdot\|_{\tau_{t}, \partial \mathcal{T}_{h}}$, we have that

$$
\begin{aligned}
\|(\boldsymbol{\Pi} \boldsymbol{\zeta}-\boldsymbol{\zeta}, \boldsymbol{\Pi} \boldsymbol{\eta}-\boldsymbol{\eta})\|_{\tau_{t}, \partial \mathcal{T}_{h}} \leq & \max _{K \in \mathcal{T}_{h}} h_{T}^{1 / 2}\left\|\tau_{t}^{-1}\right\|_{L^{\infty}(\partial T)}^{1 / 2}\left(\sum_{T \in \mathcal{T}_{h}} h_{T}^{-1}\|\boldsymbol{\Pi} \boldsymbol{\zeta}-\boldsymbol{\zeta}\|_{\partial T}^{2}\right)^{1 / 2} \\
& +\max _{K \in \mathcal{T}_{h}} h_{T}^{1 / 2}\left\|\tau_{t}\right\|_{L^{\infty}(\partial T)}^{1 / 2}\left(\sum_{T \in \mathcal{T}_{h}} h_{T}^{-1}\|\boldsymbol{\Pi} \boldsymbol{\eta}-\boldsymbol{\eta}\|_{\partial T}^{2}\right)^{1 / 2},
\end{aligned}
$$

the result follows by a componentwise application of Lemma A.1. This completes the proof.

\section{REFERENCES}

[1] G. A. Baker, W.N. Jureidini, and O. A. Karakashian, Piecewise solenoidal vector fields and the Stokes problem, SIAM J. Numer. Anal. 27 (1990), 1466-1485. MR1080332 (91m:65246)

[2] P. Bastian and B. Rivière, Superconvergence and $H$ (div) projection for discontinuous Galerkin methods, Internat. J. Numer. Methods Fluids 42 (2003), 1043-1057. MR1991232 (2004f:65177)

[3] J. Carrero, B. Cockburn, and D. Schötzau, Hybridized, globally divergence-free LDG methods. Part I: The Stokes problem, Math. Comp. 75 (2006), 533-563. MR2196980 (2006m:76040)

[4] B. Cockburn, Two new techniques for generating exactly incompressible approximate velocities, Computational Fluid Dynamics 2006. Proceedings of the Fourth International Conference in Fluid Dynamics, ICCDF4, Ghent, Belgium, 10-14 July 2006 (H. Deconinck and E. Dick, eds.), Springer-Verlag, 2009, pp. 1-11.

[5] B. Cockburn and J. Gopalakrishnan, Incompressible finite elements via hybridization. Part I: The Stokes system in two space dimensions, SIAM J. Numer. Anal. 43 (2005), 1627-1650. MR2182142 (2006m:65262)

[6] - Incompressible finite elements via hybridization. Part II: The Stokes system in three space dimensions, SIAM J. Numer. Anal. 43 (2005), 1651-1672. MR2182143 (2006m:65263)

[7] _ The derivation of hybridizable discontinuous Galerkin methods for Stokes flow, SIAM J. Numer. Anal. 47 (2009), 1092-1125. MR2485446 (2010d:65316)

[8] B. Cockburn, J. Gopalakrishnan, N.C. Nguyen, J. Peraire, and F.J. Sayas, Analysis of HDG methods for Stokes flow, Math. Comp. 80 (2011), 723-760. MR2772094

[9] B. Cockburn, J. Gopalakrishnan, and F.-J. Sayas, A projection-based error analysis of HDG methods, Math. Comp. 79 (2010), 1351-1367. MR2629996 (2011d:65354)

[10] B. Cockburn, G. Kanschat, and D. Schötzau, A locally conservative LDG method for the incompressible Navier-Stokes equations, Math. Comp. 74 (2005), 1067-1095. MR2136994 (2006a:65157)

[11] _ A note on discontinuous Galerkin divergence-free solutions of the Navier-Stokes equations, J. Sci. Comput. 31 (2007), 61-73. MR2304270 (2008f:76109)

[12] B. Cockburn, G. Kanschat, and D. Schötzau, An equal-order DG method for the incompressible Navier-Stokes equations, J. Sci. Comput. 40 (2009), 141-187. MR2511732 (2010i:65263)

[13] B. Cockburn and F.J. Sayas, Divergence-conforming HDG methods for Stokes flow. Submitted.

[14] M. Dauge, Stationary Stokes and Navier-Stokes systems on two- or three-dimensional domains with corners. I. Linearized equations, SIAM J. Math. Anal. 20 (1989), no. 1, 74-97. MR 977489 (90b:35191)

[15] M. Fortin, Calcul numérique des écoulements des fluides de Bingham et des fluides newtoniens incompressibles par la méthode des élements finis, Ph.D. thesis, Université de Paris VI, 1972.

[16] _ Utilization de la méthode des éléments finis en mécanique des fluides, Calcolo 12 (1975), 405-441. MR0421339 (54:9344a)

[17] D.F. Griffiths, Finite elements for incompressible flow, Math. Methods Appl. Sci. 1 (1979), 16-31. MR.548403 (80j:76027)

[18] P. Hansbo and M.G. Larson, Discontinuous finite element methods for incompressible and nearly incompressible elasticity by use of Nitsche's method, Comput. Methods Appl. Mech. Engrg. 191 (2002), 1895-1908. MR1886000(2003j:74057) 
[19] F. Hecht, Construction d'une base $P_{1}$ non conforme à divergence nulle, RAIRO Modél. Math. Anal. Numér. 15 (1981), 119-150. MR618819 (83j:65023)

[20] J.-C. Nédélec. Mixed finite elements in $\mathbf{R}^{3}$, Numer. Math.35 (1980) 315-341. MR592160 $(81 \mathrm{k}: 65125)$

[21] J.-C. Nédélec. A new family of mixed finite elements in $\mathbf{R}^{3}$. Numer. Math. 50 (1986) 57-81. MR864305(88e:65145)

[22] N.C. Nguyen, J. Peraire, and B. Cockburn, A comparison of HDG methods for Stokes flow, J. Sci. Comput. MR2679797(2011g:65246)

[23] _ A hybridizable discontinuous Galerkin method for Stokes flow, Comput. Methods Appl. Mech. Engrg. 199 (2010), 582-597.

[24] L. R. Scott and M. Vogelius, Norm estimates for a maximal right inverse of the divergence operator in spaces of piecewise polynomials, RAIRO Modél. Math. Anal. Numér. 19 (1985), 111-143. MR813691 (87i:65190)

[25] F. Thomasset, Implementation of finite element methods for Navier-Stokes equations, Springer Series in Computational Physics, Springer-Verlag, New York, 1981. MR720192 (84k:76015)

[26] J. Wang and X. Ye, New finite element methods in computational fluid dynamics by H(div) elements, SIAM J. Numer. Anal. 45 (2007), 1269-1286. MR22318812 (2009b:65328)

School of Mathematics, University of Minnesota, 206 Church Street S.E., MinneapoLis, Minnesota 55455

E-mail address: cockburn@math.umn.edu

Institute for Mathematics and Its Applications, University of Minnesota, MinneapoLis, Minnesota 55455

E-mail address: jcui@ima.umn.edu 